\title{
Low Emittance Muon Beams from Positrons
}

\author{
Francesco Collamati ${ }^{*}$, Mario Antonelli ${ }^{b}$, Manuela Boscolo ${ }^{b}$, Marica Biagini ${ }^{b}$, Oscar \\ Blanco-Garcia $^{b}$, Alessandro Variola ${ }^{b}$, Alberto Bacci ${ }^{c}$, Simone Liuzzo ${ }^{d}$, Pantaleo \\ Raimondi $^{d}$, Iryna Chaikovska ${ }^{e}$, Robert Cheab ${ }^{e}$ et Lewis Keller ${ }^{f}$ \\ ${ }^{a} I N F N$ - Rome, ${ }^{b} I N F N$ - LNF, INFN - Milan, ${ }^{d}$ ESRF - Grenoble, ${ }^{e}$ LAL - Orsay, ${ }^{f}$ SLAC - Stanford, ${ }^{h}$ \\ CERN - Geneve, \\ E-mail: francesco.collamati@romal.infn.it, \\ manuela.boscolodlnf.infn.it, marica.biagini@lnf.infn.it, \\ oscar.blancodlnf.infn.it, alessandro.varioladlnf.infn.it, \\ alberto.bacci@mi.infn.it, simone.liuzzodesrf.fr, \\ pantaleo.raimondidesrf.fr, chaikovselal.in2p3.fr, \\ chehabelal.in2p3.fr, kellereslac.stanford.edu,
}

\begin{abstract}
We are studying a novel scheme to produce muon beams characterised by very low emittance, thus allowing to avoid the need for cooling, using a positron beam of about $45 \mathrm{GeV}$ interacting on electrons on a fixed target. This is a challenging scheme, and a full design study has to be developed. One of the key innovative topics to be investigated regards the interaction between the positron beam stored in a low emittance ring with a thin target inserted directly in the ring chamber. Produced muons will then be immediately collected at the exit of the target and transported to two $\mu^{+}$and $\mu^{-}$accumulator rings. In this paper, after an introduction highlighting the rational in designing a muon collider, we discuss in detail this new muon production scheme, covering the simulation of the e+ beam interacting with the target, its degradation in the 6-D phase space and the optimisation of the e+ ring design mainly to maximise the energy acceptance.
\end{abstract}

The 19th International Workshop on Neutrinos from Accelerators NUFACT2017

25-30 September 2017

Uppsala University, Uppsala, Sweden

\footnotetext{
*Speaker.
} 


\section{Introduction}

The first proposals of a muon collider date back to several decades ago. However, such a hypothetical machine is gaining more and more interest today as a possible new generation of experiment, capable of overtaking the principal limitations of today's colliders, such as LHC. In fact, even if the Large Hadron Collider has yet to deliver several years of data, given the great effort needed to conceive and build such frontier machines it is already now the time to discuss how to carry on the research on fundamental particle physics in the years to come.

In this context, design studies are currently ongoing on a Future Circular Collider, to be build at CERN, with a circumference of about $100 \mathrm{~km}$ and unprecedented energies and luminosities for both hadron and lepton alternatives. However, even with respect to such huge and powerful machines, a muon collider would still mantein some advantages that make it worth studying.

The vast part of these advantages comes from the use of muons itself. In fact, being 200 times heavier than electrons, a beam of muons will suffer much less of Synchrotron Radiation emission, that is the limit of circular $e^{+} e^{-}$colliders, giving the possibility to reach much higher energies. Moreover, the lack of light emission also allows to obtain much smaller energy spread of the beam, resulting in a much better energy resolution.

The advantages, however, are not only related to the accelerator, but spread also to the physics itself. In fact, focusing for example on the Higgs boson, since its coupling goes with $m^{2}$, we expect a much higher production of Higgs boson at a muon collider.

On the other hand, in spite of these advantages there are also few critical points that have hindered so far the design of a muon collider. First and foremost, muons decay in few $\mu$ s. As a result, the whole chain, from generation to acceleration up to the interaction, must be performed very quickly. The other main limitation is related to the traditional muon production scheme, that leads inevitably to large emittance beams. In fact, muons are conventionally produced by colliding protons on target, and then exploiting the $\pi / k$ decays [1] [2]. Muons are thus created with a variety of angles and energies, thus calling for beam cooling to lower its emittance.

In this paper, we present a new scheme to produce muon beams characterised by intrinsic low emittance, that would allow to avoid the need for cooling.

\section{Direct muon production}

In this novel scheme, a primary beam of $45 \mathrm{GeV}$ positrons interacts with a fixed target, this energy being just above the threshold for the reaction $e^{+} e^{-} \rightarrow \mu^{+} \mu^{-}$[3] [4] [5] [6]. The crucial advantage of this "direct muon production" is that it allows to obtain low emittance muons. In fact, the angle between the 2 muons can be shown to go with $\sqrt{s}$, being however small close to the threshold. Moreover, also the energy difference between the two muons produced is small just above the threshold, thus implying that also the energy spread of the muon beam will be small. As a result, exploiting direct muon production it is possible to create a muon beam that has since from creation small emittance, implying that good luminosity is reachable with reduced muon fluxes, thus resulting in reduced backgrounds. Lastly, also the losses from decays are strongly reduced. In fact, this asymmetric scheme allows to obtain two muons that are already boosted, which will 
thus have longer lifetime and which are both possible to collect, actually doubling the production efficiency.

However, on the other side with respect to such advantages, the main limitation of this novel scheme is in the production rate, since the cross section for the process is about 3 orders of magnitude smaller than the one for the conventional one ( $\mu b$ vs $m b$ ).

Such a small cross section makes the choice of the target material crucial. In fact, the number of produced $\mu$ pairs $\left(N_{\mu \mu}\right)$ can be parametrised as:

$$
N_{\mu \mu}=N_{e^{+}} \rho_{e^{-}} L \sigma_{\left(e^{+} e^{-} \rightarrow \mu^{+} \mu^{-}\right)},
$$

where $N_{e^{+}}$is the number of positrons hitting the target, $\rho_{e^{-}}$is the electronic density of the target, $L$ is the length of the target, and $\sigma$ the cross section of the production process.

Therefore, the criteria to follow to identify the ideal target are basically 3: first of all, a thin target is needed in order to minimise the emittance. In fact, a thick target would mean that muons are produced all along its thickness, thus resulting in an increase in beam emittance. Secondly, a high density target is needed to maximise the production rate. Lastly, the positron loss should be as much reduced as possible, since to rise the number of produced muons is it possible to conceive a recirculating scheme in which the positron beam interacts several times in the target. This last point would therefore require a low $\mathrm{Z}$ target, in order to limit the effect of bremsstrahlung and Bhabha scattering, that would reduce the positron beam lifetime.

It is thus evident that these requirements are somehow conflicting with each other. Consequently, a careful study relying on Monte Carlo simulation is being performed. In principle, a possible tradeoff between the conflicting requirements could be represented by not too heavy materials (like $\mathrm{Be}, \mathrm{C}$ and $\mathrm{Li}$ ) and not too thin targets.

Nevertheless, in order to maximise the production rate, the other fundamental aspect to be considered is the positron source, that has to be as intense as possible $\left(\sim 10^{18} e^{+} / s\right)$.

\section{Accelerator scheme}

The current proposal for the accelerator scheme is shown in Fig. 1 (left), and starts from a standard electron gun, whose electrons collide with a conventional Heavy Target (HT) to produce $e^{+} e^{-}$pairs. Then, an Adiabatic Matching Device is used to collect positrons, that are accelerated and injected in the main positron ring. At the current design stage, this is a $6.3 \mathrm{~km} 45 \mathrm{GeV}$ storage ring in which a target for muon production $(\mathrm{T})$ is inserted, in such a way to make positrons collide with it several times. In this configuration, muons are produced with an energy of $22 \mathrm{GeV}$ and a Lorentz $\gamma$ factor of about 200, meaning that they will live about $500 \mu s$. In correspondence of the production target two separate muon accumulation rings are foreseen, followed by a fast acceleration line that injects both muon beams in the actual final collider.

A first set of parameters for the positron ring is shown in Fig. 1 (center), together with a very preliminary set of parameters for a possible muon collider exploiting the proposed scheme. However, despite being these very preliminary results, what is worth to stress is that thanks to the very small emittance a luminosity comparable to the conventional one is possible with much lower muon fluxes. 


\subsection{Positron ring optics scheme}

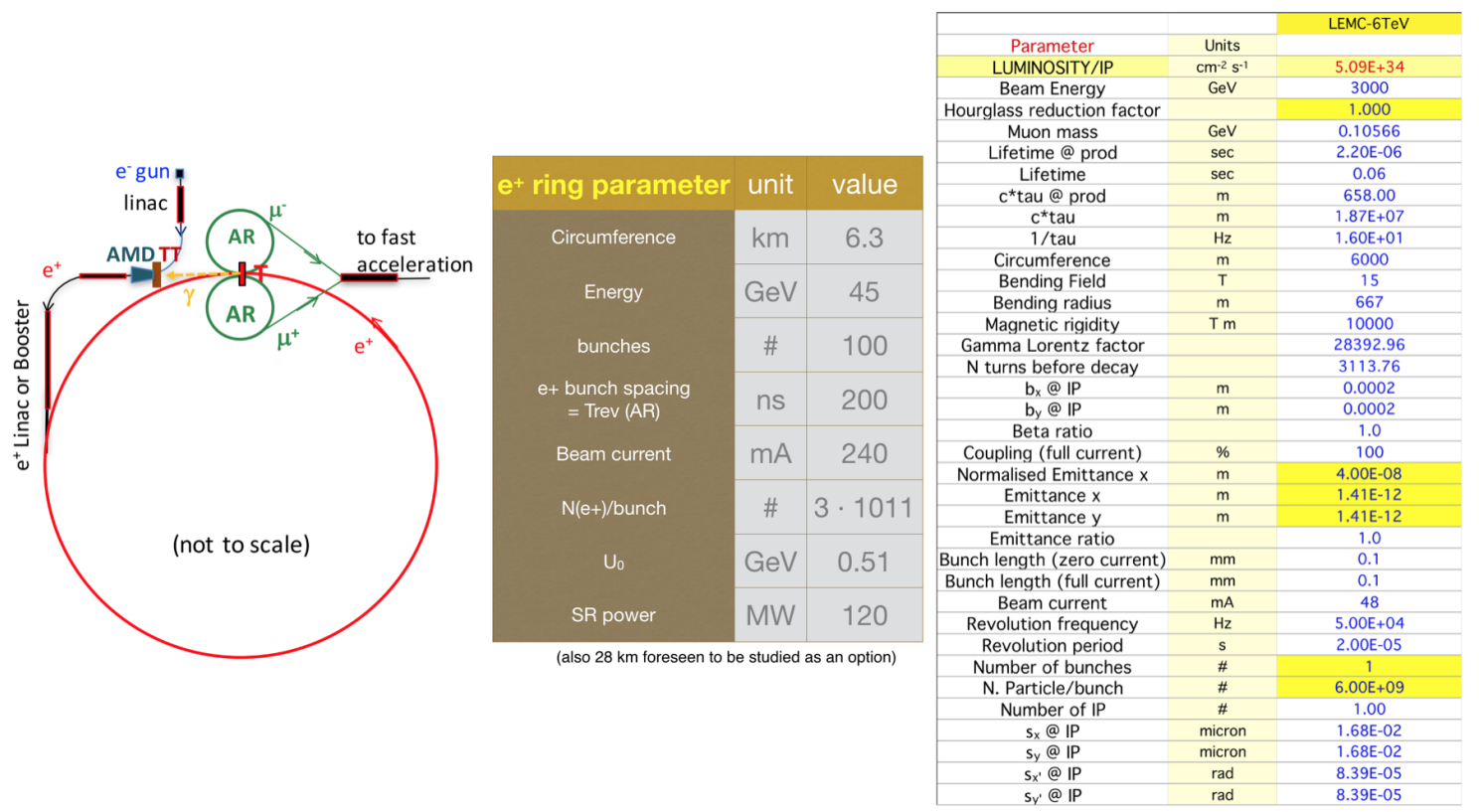

Figure 1: Left: Current design of the accelerator complex, as described in the text. Center: set of parameters for the positron ring. Right: set of preliminary parameters for a possible muon collider exploiting the described scheme.

A full optical design for the positron ring has been produced, consisting in a series of $200 \mathrm{~m}$ cells, with a total length of $6.3 \mathrm{~km}$. The beta functions and momentum acceptance for the cell are being studied both Accelerator Toolbox [7] and MAD-X PTC [8], finding good agreement between the two codes, and are shown in Fig. 2 (left). Some more details on the machine are reported in the table in Fig. 2 (right).

\subsection{Low $\beta$ interaction region insertion}

In order to minimise the degradation produced by the target and maximise the muon production, the ideal goal for this machine would be to have a zero dispersion and low beta Interaction Region in which to place the muon production target. Up to now, the optimisation of the lattice allowed to reach these values at the target position:

$$
\beta_{x}=1.6 m ; \beta_{y}=1.7 m ; D_{x}=5.4 m m .
$$

In Fig. 3 the dynamic and momentum aperture corresponding to this values are shown, highlighting the worsening effect of adding the Insertion Region in the cell. However, also in this context further optimisation are conceivable, in particular attempting to match the transverse minimum beam size with constraints of target thermo-mechanical stress, that put limits on the size of the beam impinging on it. Also dynamic and momentum aperture can still be optimised.

\subsection{Positron source simulation}

One of the key challenges to be addressed in order to develop the here proposed machine is the positron source, that has to be as intense as possible to limit the effect of the low efficiency of 


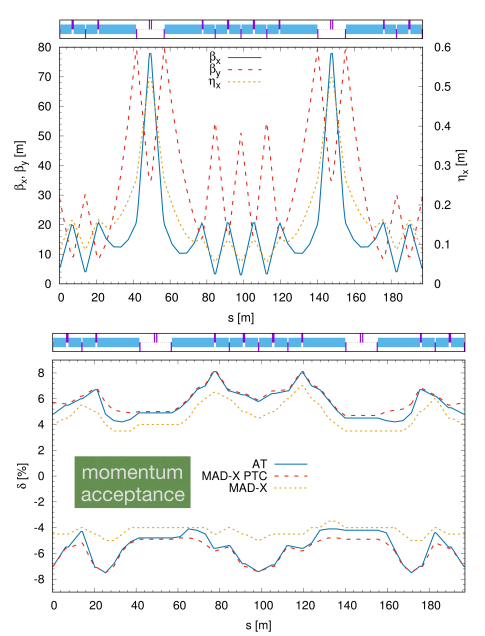

\begin{tabular}{lll}
\hline \hline Parameter & Units & \\
Energy & $\mathrm{GeV}$ & 45 \\
Circumference & $\mathrm{m}$ & 6300 \\
Coupling(full current) & $\%$ & 1 \\
Emittance x & $\mathrm{m}$ & $5.73 \times 10^{-9}$ \\
Emittance y & $\mathrm{m}$ & $5.73 \times 10^{-11}$ \\
Bunch length & $\mathrm{mm}$ & 3 \\
Beam current & $\mathrm{mA}$ & 240 \\
RF frequency & $\mathrm{MHz}$ & 500 \\
RF voltage & $\mathrm{GV}$ & 1.15 \\
Harmonic number & $\#$ & 10508 \\
Number of bunches & $\#$ & 100 \\
N. particles/bunch & $\#$ & $3.15 \times 10^{11}$ \\
Synchrotron tune & & 0.068 \\
Transverse damping time & $\mathrm{turns}$ & 175 \\
Longitudinal damping time & turns & 87.5 \\
Energy loss/turn & $\mathrm{GeV}$ & 0.511 \\
Momentum compaction & & $1.1 \times 10^{-4}$ \\
RF acceptance & $\%$ & \pm 7.2 \\
Energy spread & $\mathrm{dE} / \mathrm{E}$ & $1 \times 10^{-3}$ \\
SR power & $\mathrm{MW}$ & 120 \\
\hline \hline & & \\
\hline
\end{tabular}

Figure 2: Twiss functions (left, top) and momentum acceptance (left, bottom) of the positron ring cells, studied with MAD-X PTC and Accelerator Toolbox, and a table (right) with some parameters of the lattice.
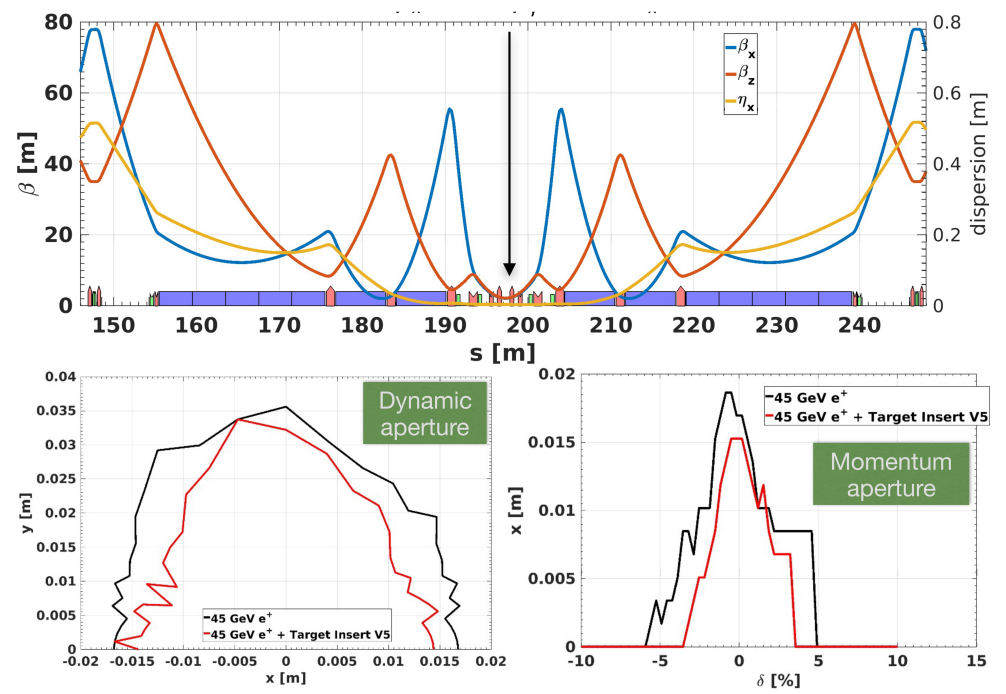

Figure 3: Top: Beta functions and Dispersion when adding the target to te cell. Bottom: dynamic (left) and momentum (aperture) of the cell when inserting the target.

the production process. Moreover, the LEMMA scheme deeply relies on the possibility to enhance the muon production by recirculating the positron beam, making it interact several time with the target. However, at each interaction with the target, positrons will loose part of their energy, and some of them will eventually be lost.

Nevertheless, it is possible to conceive a way to recover this loss rate exploiting the production of secondary positrons in a second heavy target due to pair production of photons created by Bremsstrahlung in the interaction of the primary beam on the target [9]. This scheme is sketched in Fig. 4, top. A full GEANT4 [10] simulation comprehending both the primary and the secondary target has been developed in order to assess the efficiency of this technique, in terms of number and 
quality (energy and angular spread) of the created positrons.

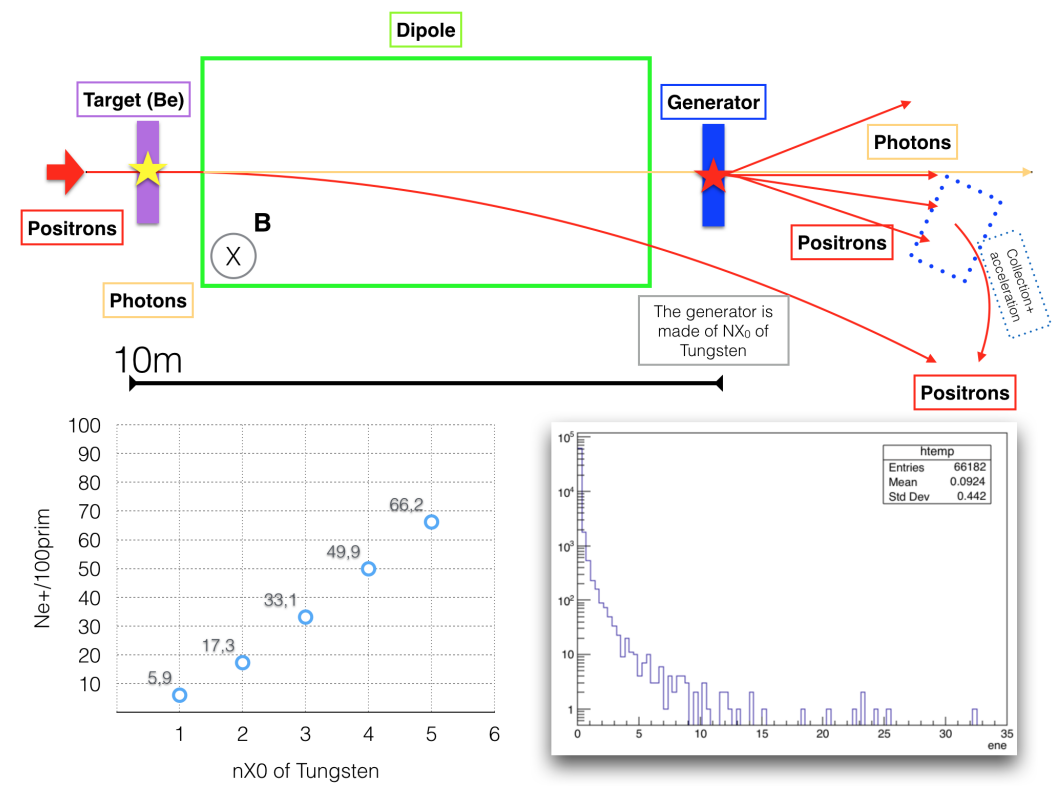

Figure 4: Top: Proposed scheme for production of secondary positrons. Bottom, left: number of positrons created in the secondary Tungsten target as a function of its thickness. Bottom, right: energy spectrum of positrons exiting the secondary target of $5 X_{0}$ of Tungsten.

Fig. 4 bottom, shows that in case of $5 X_{0}$ thick Tungsten secondary target about 66 secondary positrons are created per 100 primary positrons. Since the same GEANT4 simulation suggests that when traversing the primary target about $3 \%$ of positrons fall outside the energy acceptance of the machine, it would be in principle sufficient to have a collection efficiency of about $5 \%$ to recover enough secondary positrons to compensate the loss in the primary beam.

The choice of thickness and material of the generator is however not trivial, since also for this second target there are thermo mechanical constraints that could suggest to choose a thinner target, if the collection efficiency should be enough. Studies regarding the collection of this secondary positrons are already ongoing, relying on an ASTRA simulation.

\section{Multi turn simulation studies}

One of the main peculiarity of the proposed machine is the multiple interactions of the primary beam within the target. Therefore, a dedicated multi turn simulation scheme has been set up, in order to follow the evolution of the beam at every interaction in the target and to optimise its parameters. The simulation starts with the initial 6D beam distribution, taken from the equilibrium emittances. This distribution is tracked for a whole turn up to the target using both Accelerator Toolbox and MAD-X PTC for comparison. Then, the behaviour of the positrons inside the target is simulated by means of FLUKA [11] [12] and GEANT4Monte Carlo codes, which exit distribution is sent back to the tracking program until the next interaction in the target.

So, at each pass through the target the positron beam undergoes two processes. First of all, it gets an angular kick due to Multiple Scattering, leading to an increase in the emittance. Secondly, 
positrons loose energy due to bremsstrahlung, and thus the role of momentum acceptance of the positron ring becomes crucial in order not to loose them. The number of particles surviving at each turn in shown in Fig. 5.

Moreover, by means of this multi turn simulation it is also possible for example to study the variation of the positron beam lifetime as a function of the target thickness and material. It turns out that the process that dominates the loss is radiative interaction in the target, rather than Multiple Scattering.

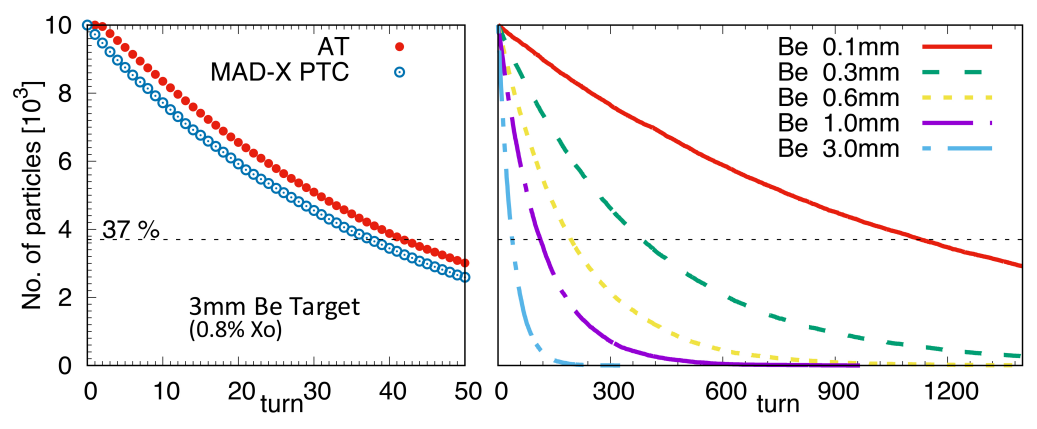

Figure 5: Top: fraction of particles surviving as a function of number of turns for several thicknesses of Beryllium target (left: zoom for $3 \mathrm{~mm}$ Be case). Bottom left: contribution to particle losses for the two processes. Bottom right: beam lifetime as a function of target thickness.

Furthermore, this multi turn simulation technique allows to evaluate the increase in beam size as a function of turns, also factorising the contribution coming from bremsstrahlung and Multiple Scattering to this emittance increase. It turns out that the dominating contribution in the current machine design is given by bremsstrahlung, that however is expected to become almost negligible once a 0 dispersion interaction region will be available.

\section{Conclusion}

In this paper we described an innovative approach to muon production, that could allow the design of a muon collider characterised by very low emittance without the need of cooling. The main limitation of this new production scheme is the low rate due to the small cross section of the process of interest. To overcome it, among other things it is crucial to optimise the target choice, in such a way to maximise the number of muons created, resulting in the need to sustain a high radiation load.

A first design of the low emittance positron ring has been developed and presented in this paper, with preliminary studies of beam dynamics, also exploiting a multi turn simulation that has been specifically developed joining tracking codes like MAD-X PTC or Accelerator Toolbox with Monte Carlo codes like FLUKA and GEANT4.

Even if there is still plenty of room for optimisation in several aspects, from the machine to the target to the positron source needed, the first results shown in this paper seems to be promising. 


\section{References}

[1] Muon Accelerator Program web page: http://map.fnal.gov

[2] M.A. Palmer, The US muon accelerator program, Proceeding of IPAC2014 - Dresden, Germany (TUPME012)

[3] M. Antonelli, M. Boscolo, R. Di Nardo, P. Raimondi, Novel proposal for a low emittance muon beam using positron beam on target, Nuclear Instruments and Methods in Physics Research A 807 (2016) 101-107

[4] M. Antonelli, M. Biagini, M. Boscolo, A. Variola, P. Raimondi, G. Cavoto, E. Bagli, Very low emittance muon beam using positron beam on target, Proceeding of IPAC2016 - Busan, Corea (TUPMY001)

[5] M. Boscolo, M. Antonelli, M. Biagini, O. Blanco-Garcia, A. Variola, F. Collamati, A. Bacci, S. Liuzzo, P. Raimondi, I. Chaikovska, R. Chehab, M. Iafrati, L. Keller, Studies of a scheme for low emittance muon beam production from positrons on target, Proceeding of IPAC2017 - Copenhagen, Denmark (WEOBA3)

[6] W. A. Barletta and A. M. Sessler, Characteristics of a high-energy $\mu^{+} \mu^{-}$collider based on electroproduction of muons, Nuclear Instruments and Methods in Physics Research A 350 (1994) 36

[7] B. Nash et al., New Functionality for Beam Dynamics in Accelerator Toolbox (AT), Proceeding of IPAC2015 - Richmond, VA, USA (MOPWA014)

[8] MAX-X web page: http://madx.web.cern.ch/madx/.; E. Forest and F. Schmidt, PTC - User Reference Manual (2010).

[9] R. Chehab, Angular collection using solenoids, Nuclear Instruments and Methods in Physics Research A 451 (2000) 362-366

[10] S.Agostinelli, et al.,GEANT4:ASimulationtoolkit, Nuclear Instruments and Methods in Physics Research A 506 (2003) 250?303.

[11] T.T. Böhlen, F. Cerutti, M.P.W. Chin, A. Fassò, A. Ferrari, P.G. Ortega, A. Mairani, P.R. Sala, G. Smirnov and V. Vlachoudis, The FLUKA Code: Developments and Challenges for High Energy and Medical Applications, Nuclear Data Sheets 120, 211-214 (2014)

[12] A. Ferrari, P.R. Sala, A. Fasso', and J. Ranft, FLUKA: a multi-particle transport code, CERN-2005-10 (2005), INFN/TC05/11, SLAC-R-773 